Research Paper

\title{
Establishment and Characterization of the Reversibly Immortalized Mouse Fetal Heart Progenitors
}

\author{
Mi Li1,2, Yuan Chen ${ }^{1}$, Yang Bi1,2, Wei Jiang2, Qing Luo ${ }^{1,2}$, Yun $\mathrm{He}^{1,2}$, Yuxi Su1,2, Xing Liu1,2, Jing Cui ${ }^{2,3}$, \\ Wenwen Zhang2,3, Ruidong Li2,3, Yuhan Kong2,3, Jiye Zhang2,3, Jinhua Wang2,3, Hongyu Zhang2,3, Wei

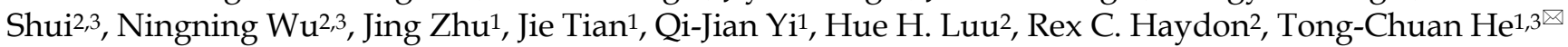 \\ and Gao-Hui Zhu' ${ }^{\text {四 }}$ \\ 1. Stem Cell Biology and Therapy Laboratory, the Key Laboratory of Pediatrics Designated by Chinese Ministry of Education and Chongqing Bu- \\ reau of Education, and the Children's Hospital of Chongqing Medical University, Chongqing 400014, China \\ 2. Molecular Oncology Laboratory, Department of Orthopaedic Surgery, The University of Chicago Medical Center, Chicago, IL 60637, USA \\ 3. Key Laboratory of Diagnostic Medicine designated by Chinese Ministry of Education, and The Affiliated Hospitals of Chongqing Medical Uni- \\ versity, Chongqing 400016, China
}

\begin{abstract}
$\triangle$ Corresponding authors: T.-C. He, MD, PhD. Stem Cell Biology and Therapy Laboratory, The Pediatric Research Institute, The Children's Hospital of Chongqing Medical University, Chongqing 400014, China. Tel/Fax: 86-23-6363-1938. E-mail: tche@uchicago.edu. Gao-Hui Zhu, MD, PhD. Stem Cell Biology and Therapy Laboratory, The Pediatric Research Institute, The Children's Hospital of Chongqing Medical University, Chongqing 400014, China. Tel/Fax: 86-23-6363-1938. E-mail: gaohui.zhu@gmail.com
\end{abstract}

(c) Ivyspring International Publisher. This is an open-access article distributed under the terms of the Creative Commons License (http://creativecommons.org/ licenses/by-nc-nd/3.0/). Reproduction is permitted for personal, noncommercial use, provided that the article is in whole, unmodified, and properly cited.

Received: 2013.05.07; Accepted: 2013.06.09; Published: 2013.06.20

\begin{abstract}
Objective: Progenitor cell-based cardiomyocyte regeneration holds great promise of repairing an injured heart. Although cardiomyogenic differentiation has been reported for a variety of progenitor cell types, the biological factors that regulate effective cardiomyogenesis remain largely undefined. Primary cardiomyogenic progenitors (CPs) have a limited life span in culture, hampering the CPs' in vitro and in vivo studies. The objective of this study is to investigate if primary CPs isolated from fetal mouse heart can be reversibly immortalized with SV40 large T and maintain long-term cell proliferation without compromising cardiomyogenic differentiation potential.

Methods: Primary cardiomyocytes were isolated from mouse EI5.5 fetal heart, and immortalized retrovirally with the expression of SV40 large T antigen flanked with loxP sites. Expression of cardiomyogenic markers were determined by quantitative RT-PCR and immunofluorescence staining. The immortalization phenotype was reversed by using an adenovirus-mediated expression of the Cre reconbinase. Cardiomyogenic differentiation induced by retinoids or dexamethasone was assessed by an $\alpha$-myosin heavy chain $(\mathrm{MyHC})$ promoter-driven reporter.

Results: We demonstrate that the CPs derived from mouse EI5.5 fetal heart can be efficiently immortalized by SV40 T antigen. The conditionally immortalized CPs (iCPI5 clones) exhibit an increased proliferative activity and are able to maintain long-term proliferation, which can be reversed by Cre recombinase. The iCPI5 cells express cardiomyogenic markers and retain differentiation potential as they can undergo terminal differentiate into cardiomyctes under appropriate differentiation conditions although the $\mathrm{iCPI} 5$ clones represent a large repertoire of CPs at various differentiation stages. The removal of SV40 large T increases the iCPs' differentiation potential. Thus, the iCPs not only maintain long-term cell proliferative activity but also retain cardiomyogenic differentiation potential.

Conclusions: Our results suggest that the reported reversible SV40 T antigen-mediated immortalization represents an efficient approach for establishing long-term culture of primary cardiomyogenic progenitors for basic and translational research.
\end{abstract}

Key words: Cardiomyogenic progenitors, cardiomyogenic differentiation, cardiomyogenesis, cardiovascular disorders, heart progenitor cells, immortalization 


\section{INTRODUCTION}

The use of artificial heart device and/or cardiac transplantation is currently the treatment of choice for end-stage heart failure. It is however hampered by a severe shortage of donor organs and by the danger of organ rejection. A progenitor cell-based therapy may hold promise in improving the clinical management of many cardiomyogenic disorders, such as heart failure and acute myocardial infarction (MI) [1, 2]. However, stem cell therapy approach is currently limited by the availability of transplantable cardiomyocyte progenitors (CPs). Transplantation of non-cardiomyogenic progenitors, such as skeletal muscle myoblasts and smooth muscle cells, has been proposed as an alternative therapy [2-5], which is however complicated by the fact that transplanted cells may have abnormal electrical coupling and may cause either conduction blocks or arrhythmias in vivo [2]. Other cell types/sources have also been studied to date, including bone marrow (BM)-derived mononuclear cells, hematopoietic stem cells (HSCs), and mesenchymal stem cells (MSCs), embryonic stem cells and endothelial progenitor cells [2, 6-9], some of which have shown cardiac regeneration potential in animal MI models. Although most of the clinical studies using BM-derived cells exhibit no significant side effects, stem cell-induced improvements in cardiac function remain modest $[2,10]$.

Effective myocardial regeneration has at least two prerequisites: the progenitor cells with cardiomyogenic potential and the biological approaches to the control of efficient cardiomyogenic differentiation. Thus, it is essential to maintain a long-term culture of the isolated cardiomyogenic progenitors in vitro in order to dissect the signaling pathways and/or growth factors that regulate cardiomyogenic differentiation. Thus, the urgent needs for effective cell-based cardiac regeneration are at least two-fold. The first is to identify and effectively expand progenitors with cardiomyogenic potential. The second is to identify factors that can promote effective cardiomyogenesis of these progenitor cells. Recent studies indicate that populations of cardiac progenitor cells may reside in the heart itself $[2,11,12]$. In fact, human cardiomyocyte progenitors (hCPs) have been isolated from fetal hearts [13]. These cells express the stem cell markers stem cell antigen-1 (Sca-1)-like protein and islet-1 (Isl-1), and the early cardiac transcription factors GATA-4 and Nkx2.5. These cells are capable of differentiating into spontaneously beating cells, when stimulated with the DNA methyltransferase inhibitor 5-azacytidine [13]. When the hCPs were transplanted into infarcted mouse myocardium differentiated into both cardiomyocytes and vascular cells, and that
hCP-treated animals displayed less deterioration of cardiac function in the long term compared to control mice [14]. Nonetheless, the stem cell-based myocardial regeneration is still an inefficient process with varied outcomes. Regardless of the source of the CPs, one of the major limitations is the limited life span of the isolated progenitor cells. It is critical to investigate the molecular events that regulate heart development if stem cells are to become an effective source of cardiomyocytes and the progenitors can be used for studying cardiac physiology and eventually developing cell-based therapies for heart repair. Thus, potential efficient isolation and long-term maintenance of cardiomyogenic progenitor cells are essential to the realization of stem cell therapy in myocardial disorders.

Here, we investigate if mouse CPs derived from fetal hearts can be conditionally immortalized with SV40 large $\mathrm{T}$ antigen and maintain long-term cell proliferation without compromising cardiomyogenic differentiation potential. Using the system which expresses SV40 large T antigen flanked with loxP sites, we demonstrate that mouse CPs derived from E15.5 fetal heart can be efficiently immortalized by SV40 large $\mathrm{T}$ antigen. The conditionally immortalized CPs (iCP15 clones) exhibit an enhanced proliferative activity and maintain long-term cell proliferation, which can be reversed by Cre recombinase. The iCP15 cells express most cardiomyogenic markers and retain differentiation potential as they can undergo terminal differentiate into cardiomyctes under appropriate differentiation conditions. The iCP15 clones represent a large repertoire of $\mathrm{CPs}$ at various differentiation stages. The removal of SV40 large T increases the differentiation potential of iCPs. Thus, the conditionally immortalized iCPs not only maintain long-term cell proliferation but also retain cardiomyogenic differentiation potential. Future studies should investigate the in vivo behavior of these $\mathrm{iCP} 15$ cells in a mouse model of infarcted heart. Our results suggest that the reversible immortalization strategy using SV40 large T antigen may be an efficient approach to establishing long-term cell culture of primary CPs for basic and translational research.

\section{MATERIALS AND METHODS}

\section{Cell culture and chemicals}

HEK-293 cells were obtained from the ATCC (Manassas, VA), and maintained in complete Dulbecco's modified Eagle's medium (DMEM) as described [15-19]. Mouse embryonic carcinoma line P19 was also obtained from ATCC and used as a reference 
line. Unless otherwise indicated, all chemicals were purchased from Sigma-Aldrich or Fisher Scientific.

\section{Establishment of reversibly immortalized car- diomyogenic progenitors from mouse fetal heart}

All animal work was conducted according to relevant national and international guidelines. The animal welfare, use, and care were carried out according to the guidelines and procedures that were specifically approved for this study by the Institutional Animal Care and Use Committee. E15.5 embryonic heart tissues were dissected out and rinsed with ice-cold PBS. Heart tissues were minced and digested with trypsin-EDTA in a $37^{\circ} \mathrm{C} \mathrm{CO}_{2}$ incubator with occasional agitations for 10 to $20 \mathrm{~min}$, followed by inactivating trypsin with complete DMEM containing $10 \%$ fetal bovine serum (FBS). The dissociated cells were washed with complete DMEM twice and seeded in $10-\mathrm{cm}$ cell culture dishes. At $16 \mathrm{~h}$ after plating, cells were infected with packaged retrovirus supernatant containing SSR \#69 vector [20]. The infected cells were selected with hygromycin $(0.3 \mathrm{mg} / \mathrm{ml})$ for $5 \mathrm{~d}$ and seeded into 96-well plates with serial dilutions in the presence of hygromycin. Clones grown from single cells were chosen and scaled up for further characterization. The immortalized cardiomyogenic progenitor clones are designated as iCP15 lines.

\section{Construction of $\alpha$-myosin heavy chain (MyHC) promoter-driven Gaussia luciferase (MyHC-GLuc) reporter}

A $5.5 \mathrm{~kb}$ genomic DNA fragment upstream exon 4 of the mouse $\alpha$-myosin heavy chain (MyHC) gene was PCR amplified from the $\alpha-5.5$ vector [21], and subcloned into the BamH I/Xho I sites of our homemade retroviral reporter vector $\mathrm{pBGLuc}$ to drive the expression of secreted Gaussia luciferase, resulting in pMyHC-GLuc. The reporter vector was used for transient transfection, as well as for making stable lines via retroviral infection. Cloning and construction details are available upon request.

\section{Construction of adenoviral vector expressing Cre recombinase}

Recombinant adenovirus expressing the Cre recombinase was generated using the AdEasy technology as described as previously described [22-27]. An analogous adenovirus expressing only RFP (AdRFP) was used as a control [25-27]. Details about the vector construction are available upon request.

\section{Isolation of RNA from fetal and postnatal heart tissues and cultured cells}

For isolating RNA from tissues, CD1 mouse fetal heart tissues at E13.5 and E18.5, postnatal days 1, 3, 7, and 14, and adult heart (12 weeks) tissues were harvested and crashed in liquid nitrogen. Total RNA was isolated using the TRIZOL Reagents (Invitrogen, Carlsbad, CA) according to the manufacturer's instructions. For isolating RNA from cultured cells, subconfluent cells were seeded in $75 \mathrm{~cm}^{2}$ flasks in a medium supplemented with $1 \%$ FBS with or without adenovirus infection. Total RNA was isolated using TRIZOL Reagents.

\section{Reverse transcription, quantitative real-time PCR (qPCR), and semi-quantitative RT-PCR (sqPCR) analyses}

Reverse transcriptase-PCR was carried out as

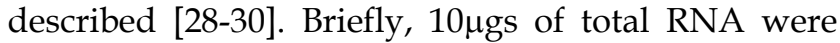
used to generate cDNA templates by reverse transcription with hexamer and M-MuLV reverse transcriptase (New England Biolabs, Ipswich, MA). The first strand cDNA products were further diluted 5- to 10 -fold and used as PCR templates. The PCR primers were 18-20mers, designed by using the Primer3 program, http://primer3.wi.mit.edu/, to amplify the 3 '-end (approximately 120-150 bps) of the gene of interest (Additional file 1: Table S1).

The qPCR was carried out as described [26, 31, 32]. SYBR Green-based qPCR analysis was carried out using the Opticon DNA Engine (Bio-Rad Laboratories, Hercules, CA). Serially diluted pUC19 was used as a standard. Duplicate reactions were carried out for each sample. All samples were normalized by endogenous level of GAPDH. Semi-quantitative RT-PCR reaction was carried out by using a touchdown protocol. PCR products were resolved on 1.5\% agarose gels. All samples were normalized by endogenous level of GAPDH.

\section{Transfection and Gaussia luciferase reporter assay}

Exponentially growing cells were seeded in $25 \mathrm{~cm}^{2}$ cell culture flasks and transfected with $2 \mu \mathrm{g}$ per flask of pMyHC-GLuc using Lipofectamine (Invitrogen). At 16h, the transfected cells were replated to 24-well plates and treated with all-trans retinoic acid (RA, final concentration $=1 \mu \mathrm{M}$; from $0.5 \mathrm{mM}$ stock prepared in DMSO) or DMSO. Gaussia luciferase possesses a natural secretory signal and upon expression is secreted into the cell medium. At the indicated time points medium from the treated cells was collected for Gaussia luciferase assays using the Gaussia Luciferase Assay Kit (New England Biolabs) as described [28-30]. Each assay condition was performed in triplicate. Reporter activity was expressed as mean \pm S.D. 


\section{Immunofluorescence staining}

Immunofluorescence staining was carried out as described [26, 31, 32]. Briefly, cells were treated with Dexamethasone (Dex, final concentration at $1 \mu \mathrm{M}$; from $0.5 \mathrm{mM}$ stock prepared in DMSO) or DMSO for $7-11$ days, and fixed with methanol at $4^{\circ} \mathrm{C}$ for $15 \mathrm{~min}$ and washed with PBS. The fixed cells were permeabilized with 1\% NP-40 and blocked with 10\% BSA, followed by incubation with a cardiac Troponin $\mathrm{T}$ (cTnT), ISL-1, or c-kit antibody (Santa Cruz Biotechnology) for $1 \mathrm{~h}$. After being washed, cells were incubated with DyLight 488 or DyLight 594-labeled secondary antibodies (Jackson ImmunoResearch Laboratories, West Grove, PA) for 30min. The presence of proteins of interest was examined under a fluorescence microscope. Stains without the primary antibody, or with control IgG, were used as negative controls.

\section{Western blotting analysis}

Western blotting was carried out as described previously [26, 31-33]. Briefly, iCP15 cells were infected with AdCre or AdGFP for 36h, lysed in Laemmli sample buffer, and subjected to SDS-PAGE. After being resolved by electrophoresis, proteins were transferred onto an Immobilon-P membrane (Millipore) via electroblotting. The membranes were blocked with SuperBlock DryBlend blocking buffer in Tris-buffered saline at room temperature for $1 \mathrm{~h}$ and probed with SV40 large T antibody for $1 \mathrm{~h}$, followed by $30 \mathrm{~min}$-incubation with a secondary antibody conjugated with horseradish peroxidase. The presence of large $\mathrm{T}$ protein was detected by using the SuperSignal West Femto chemiluminescent substrate kit. Anti- $\beta$-actin was used for a loading control.

\section{Statistical analysis}

We used the Microsoft Excel to calculate standard deviations (SD) and statistically significant differences between samples using the two-tailed Student $t$ test. A $p$-value of 0.05 was defined as statistical significance.

\section{RESULTS}

Mouse heart tissues exhibit distinct expression patterns of cardiomyogenic markers during heart development. We first examined the endogenous expression of cardiomyogenic marker genes from early embryonic stages (as early as at E13.5) to adult (12 week-old) heart tissues. The early cardiomyogenic marker ISL-1 was highly expressed at E13.5, but rapidly decreased to undetectable level at E18.5 and thereafter, while another early marker c-kit exhibited high level of expression until postnatal day 14 (Figure 1). Interestingly, the early marker Sca-1 maintained a rather high expression level in the examined time points. The expression of immediate early marker Nkx2.5 was at the highest at E13.5 and gradually decreased to a low level in adult heart tissue (Figure 1). Expression of late and mature cardiomyogenic markers connexin 43 (Cx43), $\alpha$-myosin heavy chain $(\alpha-\mathrm{MyHC})$, cardiac $\alpha$-actin (Actc1), cardiac troponin $\mathrm{T}$ (cTnT) were readily detected in all stages while their expression levels increased after birth (Figure 1). These results indicate that ISL-1, c-kit, Nkx2.5 are reliable early cardiomyogenic markers, while $\alpha$-MyHC, Actc1 and cTnT are markers for late and/or mature cardiomyocytes.

Mouse cardiomyogenic progenitor (CP15.5) cells derived from mouse E15.5 heart tissues can be effectively immortalized with SV40 large $\mathrm{T}$ antigen. To establish stable cardiomyogenic progenitors for in vitro studies of cardiomyogenesis, we isolated and cultured fetal cardiomyocytes from mouse E15.5 heart tissues (Figure 2A). The isolated cells adopted cardiomyocyte morphology and were able to form clones and maintain rhythmatic beating in culture (Additional file 2: Video-S1). To establish stable clones, the cells were infected with the packaged retroviral vector SSR \#69, which expresses SV40 large T flanked with LoxP sites (Figure 2B) [20]. The infected cells were selected in hygromycin. Single clones were isolated via serial clonal dilution of the initially infected cells. The drug-resistant stable clones, designated as the immortalized cardiomyogenic progenitor (iCP15) sublines, exhibited cardiomyocyte-like morphology (Figure 2C). More than 100 iCP15 clones were obtained. Many of the stable clones were shown to maintain rhythmatic beating during hygromycin selection although individual sublines did not exhibit the rhythmatic beating phenotype under the standard culture condition (Additional file 3: Video-S2). We also confirmed that the immortalized iPC cells were absent of hematopoietic progenitor markers, such as CD34 and CD45 (data not shown). Nonetheless, these results demonstrate that cardiomyogenic progenitors from mouse fetal hearts can be efficiently isolated and immortalized with SV40 large T antigen. 


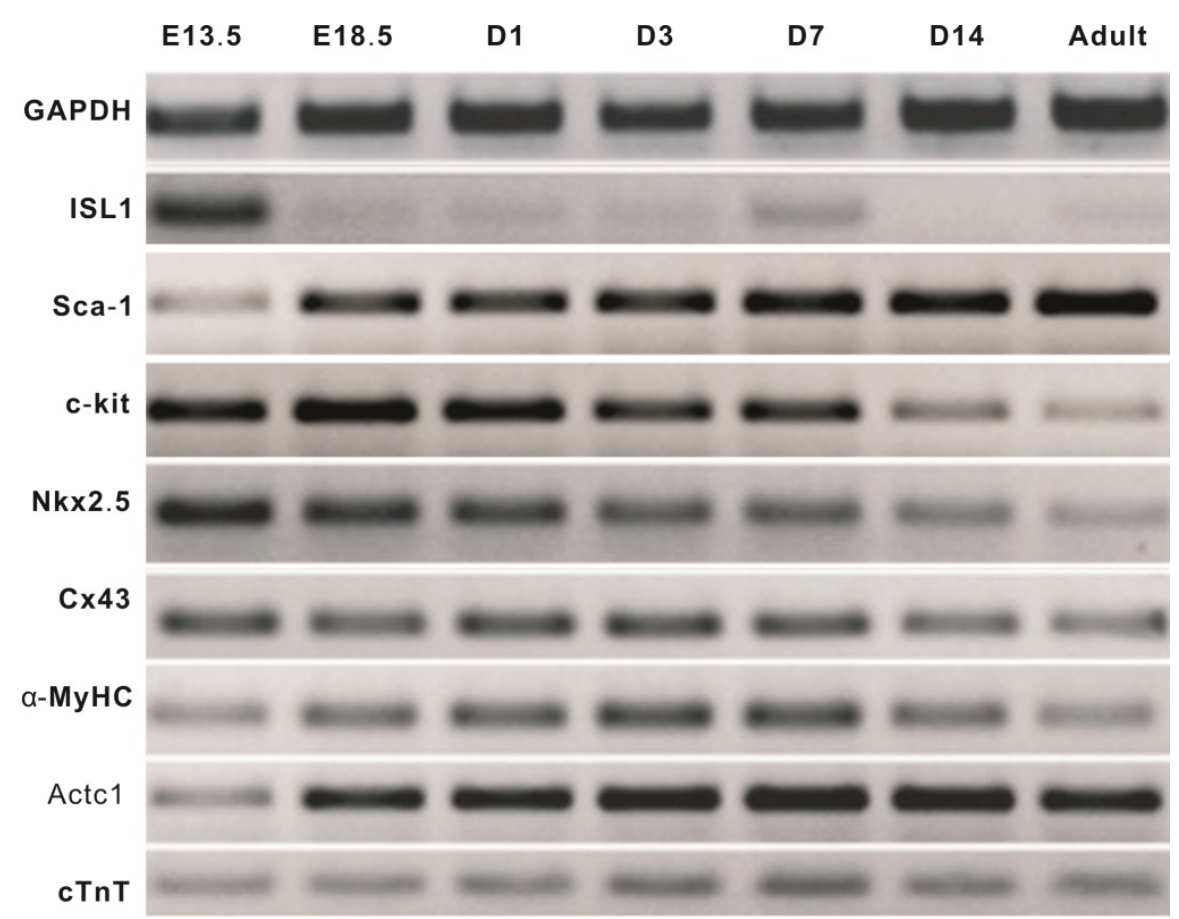

Figure I. Endogenous expression of cardiomyogenic markers during different stages of heart development. Total RNA was isolated from heart tissues as indicated and subjected to reverse transcriptase-PCR, followed by semi-quantitative RT-PCR analysis using gene-specific primers. All samples were normalized with GAPDH expression. PCR results were confirmed in at least three batches of independent experiments. Representative results are shown.
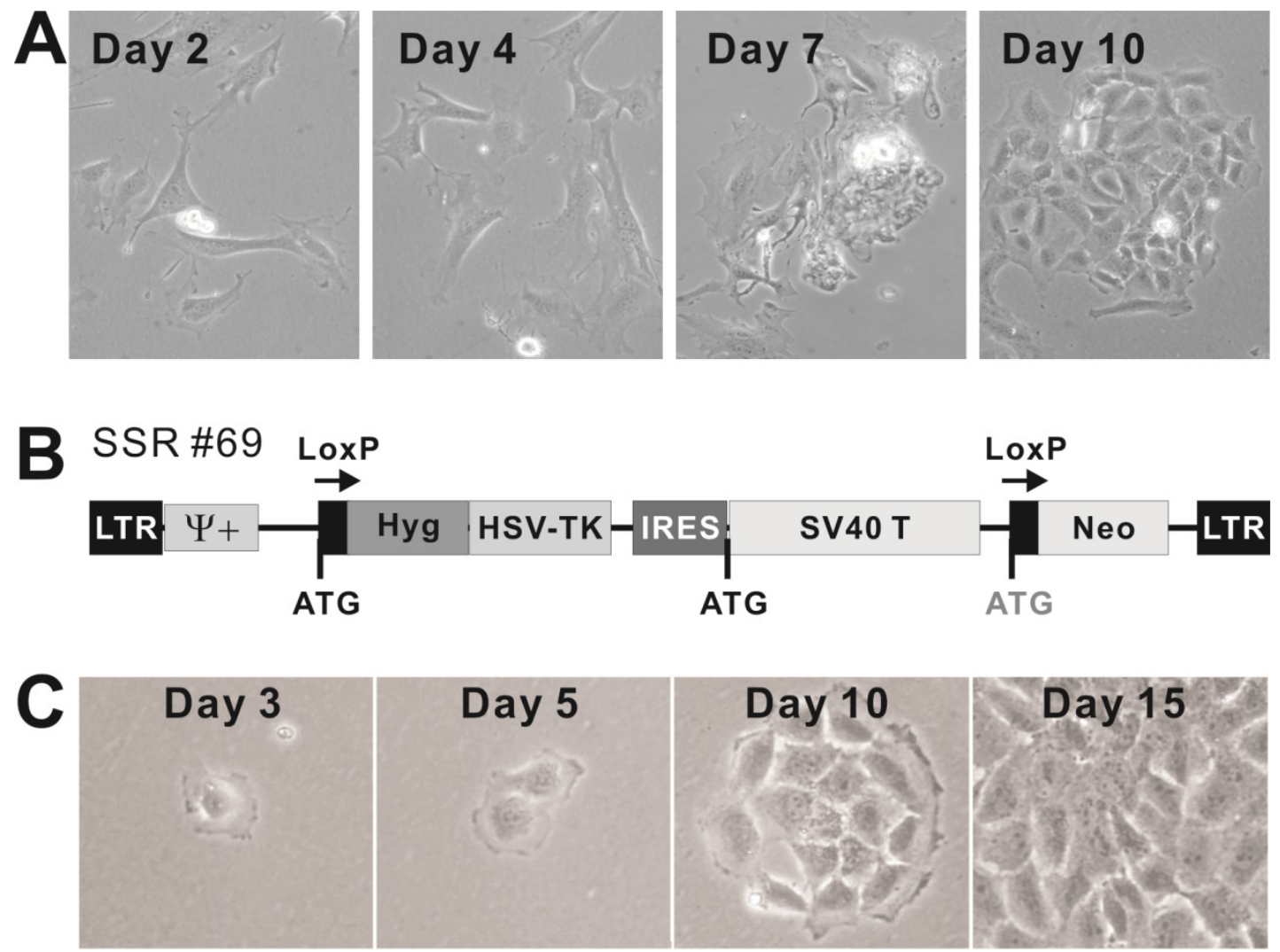

Figure 2. Isolation and immortalization of primary cardiomyogenic progenitor (CPI5.5) cells derived from mouse EI5.5 heart tissues. (A) Morphology of the isolated primary cardiomyogenic progenitors in cell culture. Representative cell clusters are shown. The primary culture cells were shown to maintain rhythmatic beating in culture (Additional file 2: Video-SI). (B) Schematic representation of the retroviral vector SSR\#69 expressing SV40 large T, which is flanked with LoxP sites, for reversible immortalization, described in [20]. (C) Establishment of immortalized CPI5.5 (iCPI5) cells. Cardiomyogenic cells were isolated from mouse EI5.5 fetal heart tissues, followed by infected with packaged retroviral vector SSR\#69. The infected cells were selected in hyrogromycin. Single clones were isolated via serial clonal dilution of the initially infected cells. Morphology of a representative drug-resistant stable is shown at different time points of selection. Some of the stable clones were shown to maintain rhythmatic beating during hyrogromycin selection (Additional file 3: Video-S2). 
The iCP15 clones represent a large repertoire of cardiomyogenic progenitors at various differentiation stages. We sought to characterize the iCP15 clones by examining the expression of stem cell and cardiomyogenic markers. Among more than 100 clones obtained, 20 iCP15 clones were chosen for initial characterization. When generic stem cell markers were assessed, 15 of the 20 clones and 18 of the 20 clones expressed high levels of Oct3/4 and Nanog, respectively, while only 4 of the 20 clones expressed a high level of Sox2 (Figure 3A). It is noteworthy that 5 clones expressed very low levels of Oct $3 / 4$ and Sox2, while clones iCP15-13 and iCP15-17 had low expression levels of all three stem cell markers (Figure 3A; Additional file 1: Figure S1A), suggesting these sublines may not represent early cardiomyogenic progenitor cells.

When most of the iCP15 clones expressed relatively high levels of Sca-1 and c-kit, only 5 clones, including iCP15-5A, iCP15-9, iCP15-10, iCP15-13 and iCP15-18, expressed the early cardiomyogenic marker ISL-1 (Figure 3B; and Additional file 1: Figure S1B). All clones and 11 of the 20 clones expressed the immediately early cardiomyogenic markers Hand 2 and Sca-1, respectively (Figure 3C), while less than one-third clones expressed the early cardiomyogenic transcription factor Nkx2.5 (Figure 3C; and Additional file 1: Figure S1C). However, most iCP15 clones expressed the cardiomyocyte-specific markers cardiac $\alpha$-actin (Actc1) and $\alpha$-myosin heavy chain $(\alpha-\mathrm{MyHC})$ (Figure 3D). Eight of the 20 clones were shown to express the late marker atrial natriuretic factor (ANF) (Figure 3D; and Additional file 1: Figure S1D). In addition, the expression of cardiomyogenic markers GATA4 and cardiac troponin T (cTnT) were detectable in most iCP15 clones while Tbx 5 was shown to express in about half of the 20 clones (Additional file 1: Figure S1D). The expression of the cardiomyogenic markers was compared to that of the mouse embryonic carcinoma line P19 (Additional file 1: Figure S1D). The semi-quantitative PCR results were further confirmed by immunofluorescence staining of the seven representative iCP15 clones using ISL-1 and c-kit antibodies (Additional file 1: Figure S2). Taken the above data together, our results suggest that, among the 20 characterized iCP15 clones, iCP15-5A, iCP15-9, iCP15-10, and iCP15-18 may represent potential cardiomyogenic progenitor cells.
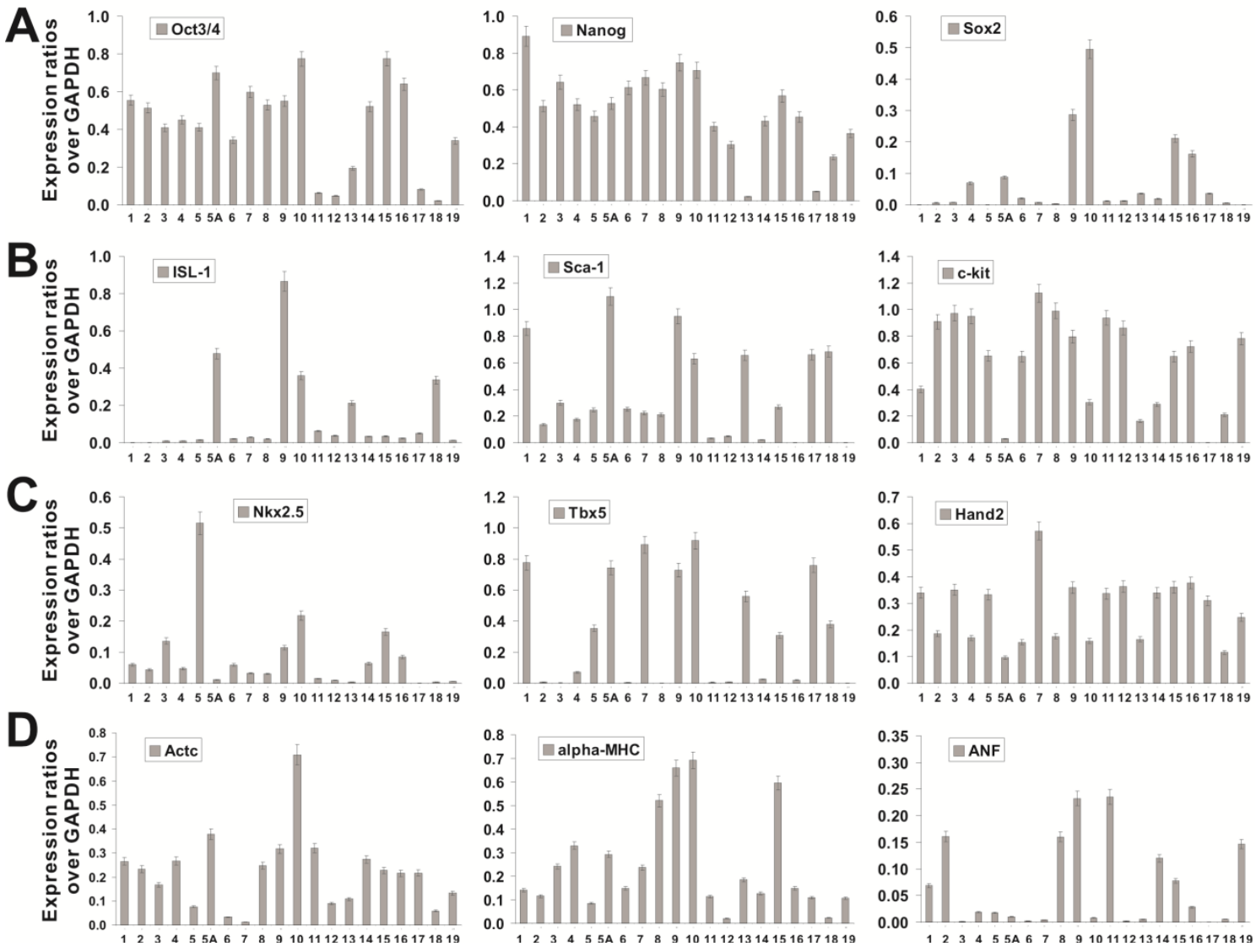

Figure 3. Characterization of $\mathrm{CPI} 5$ clones. Total RNA was isolated from 20 chosen $\mathrm{CPI} 5$ clones and subjected to reverse transcriptase reactions. The $\mathrm{cDNA}$ products were used as templates for sqPCR analysis of marker expression, including the common stem cell markers Oct3/4, Nanog, and Sox2 (A), the early cardiomyogenic progenitor markers, ISL-I, Scal and c-kit (B), the immediate early cardiomyogenic progenitor markers Nkx2.5, Tbx5, and Hand2 (C), and the late cardiomyogenic markers, cardiac $\alpha$-actin (Actcl), $\alpha$-myosin heavy chain ( $\alpha$-MyHC), and Atrial natriuretic factor (ANF) (D). All samples were normalized for their GAPDH expression. All sqPCR reactions were repeated at least in three independent experiments. Representative results are shown. 
Retinoic acid can effectively induce cardiomyogenic differentiation of the iCP15 clones. We next tested if the iCP15 clones were able to undergo cardiomyogenic differentiation upon retinoic acid stimulation. Retinoid signaling is known to regulate cardiomyogenesis [34-36]. We constructed a Gaussia luciferase reporter driven by the mouse $\alpha-\mathrm{MyHC}$ promoter (aka, MyHC-GLuc reporter) to monitor the differentiation status (Figure 4A). Eight representative iCP15 sublines were transfected with the reporter and treated with all-trans retinoic acid. We found that 6 of the 8 iCP15 lines exhibited significantly elevated fold of induction by retinoic acid, suggesting that these lines may be able to undergo cardiomyogenic differentiation (Figure 4B). All but 6 of the analyzed 20 iCP15 clones were shown to elevate MyHC-GLuc reporter activity upon RA stimulation (Figure 4C panel a). Furthermore, we analyzed the expression pattern of cardiomyogenic markers in the iCP15 clones upon RA stimulation. The expression of stem cell markers (e.g., Oct3/4 and Nanog) and early cardiomyogenic markers, such as ISL-1, Sca-1 and

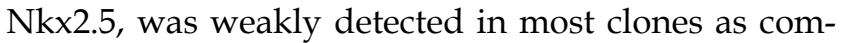
pared with that of the control group, while the expression of late markers (e.g., Actc1 and ANF) increased significantly in 4 of the 20 clones (Figure 4C panel $\mathbf{b}$ ). These results indicate that most of the tested iCP15 clones are responsive to RA-induced cardiomyogenic differentiation, and that at least four of the 20 clones (i.e., iCP15-5A, 9, 10, and 18) may undergo terminal cardiomyogenic differentiation.

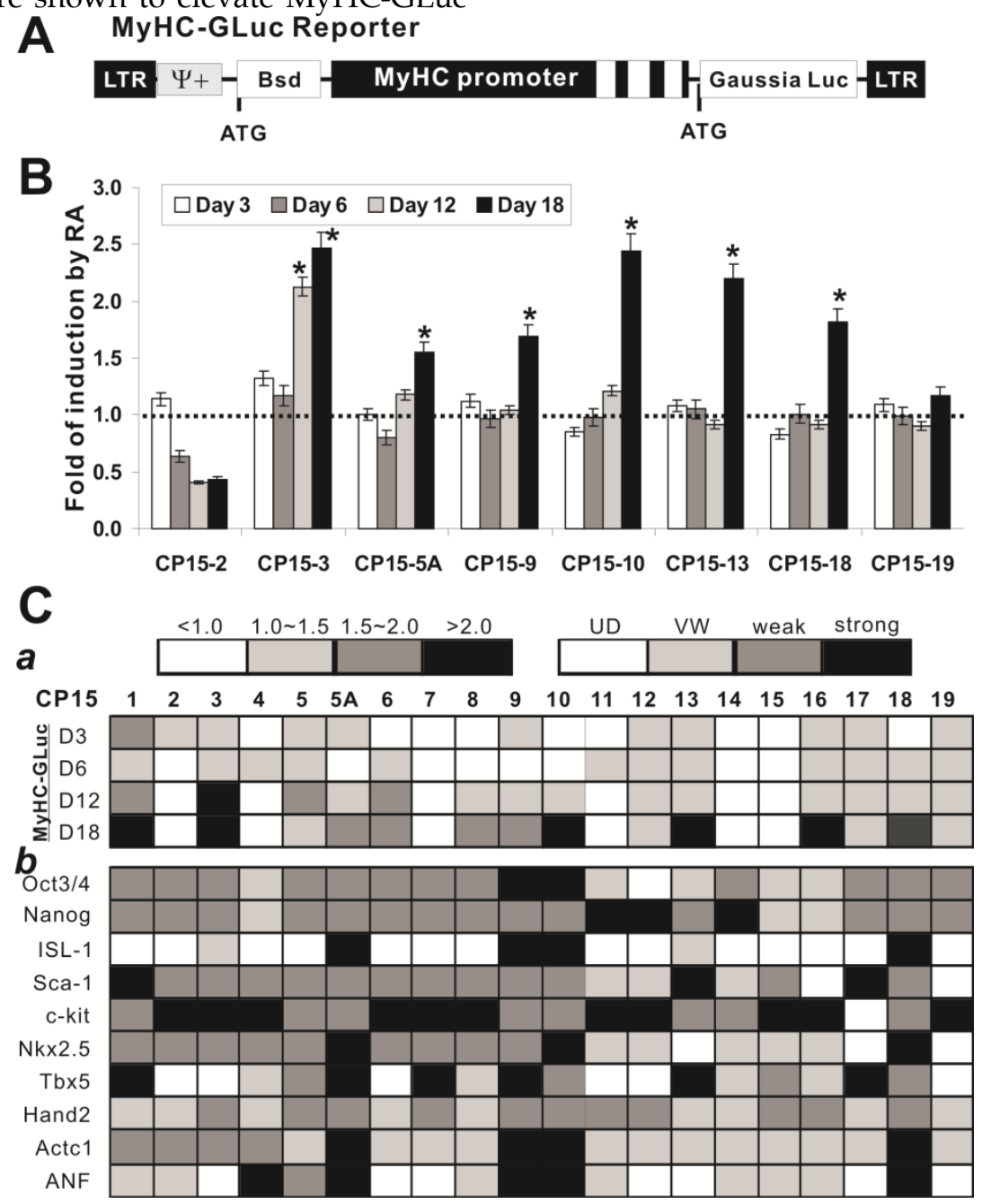

Figure 4. Characterization of the differentiation potential of iCPI 5 clones. (A) Construction of the MyHC-GLuc reporter. A 5.5kb $\alpha$-myosin heavy chain promoter-driven Gaussia luciferase reporter (MyHC-GLuc) is schematically shown. The promoter region also contains the first three exons (white boxes). (B) Retinoic acid induction of MyHC-GLuc reporter activity in iCPI5 clones. Cells of the selected eight iCPI5 clones were transfected with pMyHC-GLuc in T-25 flasks for I6-20h, replated in I2-well plates and treated with all-trans retinoic acid $(I \mu M)$ or DMSO. Culture media were collected at the indicated time points for GLuc assay (NEB). Each assay condition was carried out in triplicate. Relative GLuc activity was expressed as mean \pm SD. Fold of changes was calculated by dividing the RA-treated group with DMSO control group for each clone/time point. "*”" denotes the fold changes over control were statistically significant $(p<0.00 I)$. The dotted line represents no changes in GLuc activity between RA and control groups. (C The RA-induced differential potential and cardiomyogenic marker expression of the 20 iCPI 5 clones. (a) RA-induced MyHC-GLuc activities (fold of changes) among the 20 clones were divided into 4 categories (scale bar was shown on top left). (b) RA-induced expression levels of selected cardiomyogenic markers ware determined by sqPCR similar to that in Figure 3 and classified into 4 groups based on densitometry analysis using Imagej software (scale bar was shown on top right). 
We further tested the effect of another broad range differentiation agent Dexamethasone (Dex) on the differentiation potential of the selected iCP15 clones. Upon Dex stimulation, the expression of early marker ISL-1 decreased in 3 of the 4 selected lines, while the expression of later markers ANF, cTnT, and
$\alpha-\mathrm{MyHC}$ increased significantly (Figure 5A). The Dex-induced cTnT expression in the four iCP15 lines was further confirmed by immunofluorescence staining (Figure 5B). These results strongly suggest that the iCP15 clones may be able to undergo terminal cardiomyogenic differentiation upon Dex stimulation.

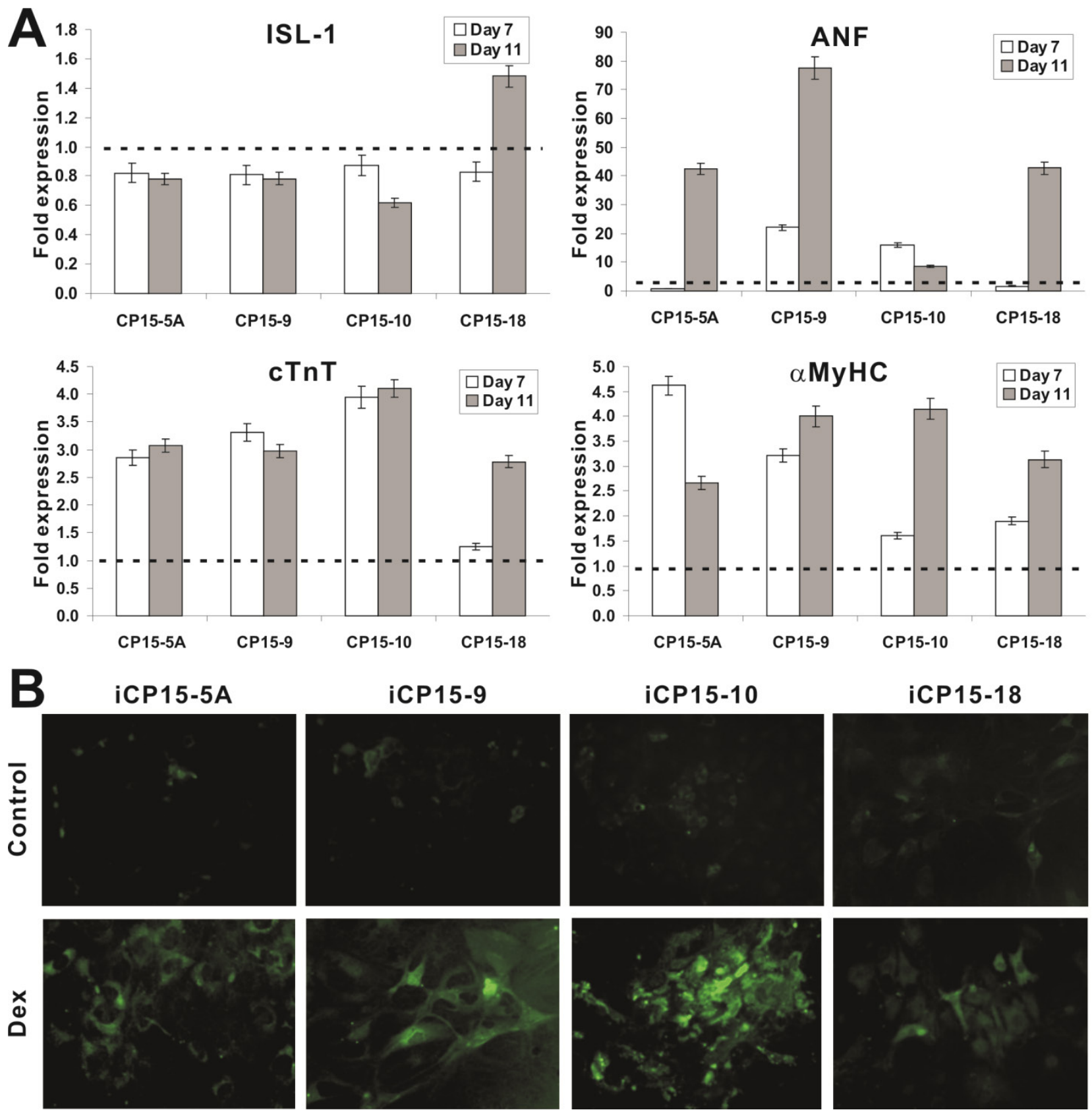

Figure 5. Cardiomyogenic differentiation potential of iCPI 5 clones. (A) Expression of cardiomyogenic markers upon in iCPI 5 cells Dex induced differentiation. The four representative iCPI5 clones were treatment with Dex $(I \mu M)$ or DMSO for 7 or II days. Total RNA was isolated and subjected to qPCR to determine ISL-I, ANF, cTnT and $\alpha-M y H C$ expression. The fold of expression was calculated by dividing Dex-treatment group with DMSO group. The dotted line indicates fold change equaling to I (or no expression changes). (B) Immunofluorescence staining of Dex-induced cTnT expression in iCPI 5 cells. The four iCPI 5 clones were treated with Dex or DMSO for 5 days and stained with anti-cTnT antibody or control IgG, followed by staining with DyLight 488-labeled secondary antibody. Representative results are shown. 
The removal of SV40 large T leads to a decreased proliferative activity of the iCP15 cells. We tested the effect of Cre recombinase-mediated removal of SV40 large $\mathrm{T}$ on the proliferation and differentiation potential of iCP15 lines. To efficiently deliver Cre recombinase into iCP15 cells, we used the previously constructed adenoviral Cre vector to transduce iCP15-5A and iCP15-9 cells [28-30]. Western blotting analysis confirmed that there was a significantly decrease in SV40 large $T$ expression in both lines (Figure 6A). The Cre-mediated removal of SV40 large T resulted in an increase in MyHC-GLuc reporter activity $(p<0.05)$
(Figure 6B), suggesting that the removal of SV40 large $\mathrm{T}$ may enhance cardiomyogenic differentiation. We further analyzed the proliferative activity of four selected clones upon SV40 large T removal and found that cell proliferation rate significantly decreased in the cells infected with Ad-Cre (Figure 6C). These results are consistent with a notation that the immortalization of the iCP15 cells can be reversed by introduction of Cre recombinase; and the removal of SV40 large $\mathrm{T}$ may promote differentiation while inhibit cell proliferation.
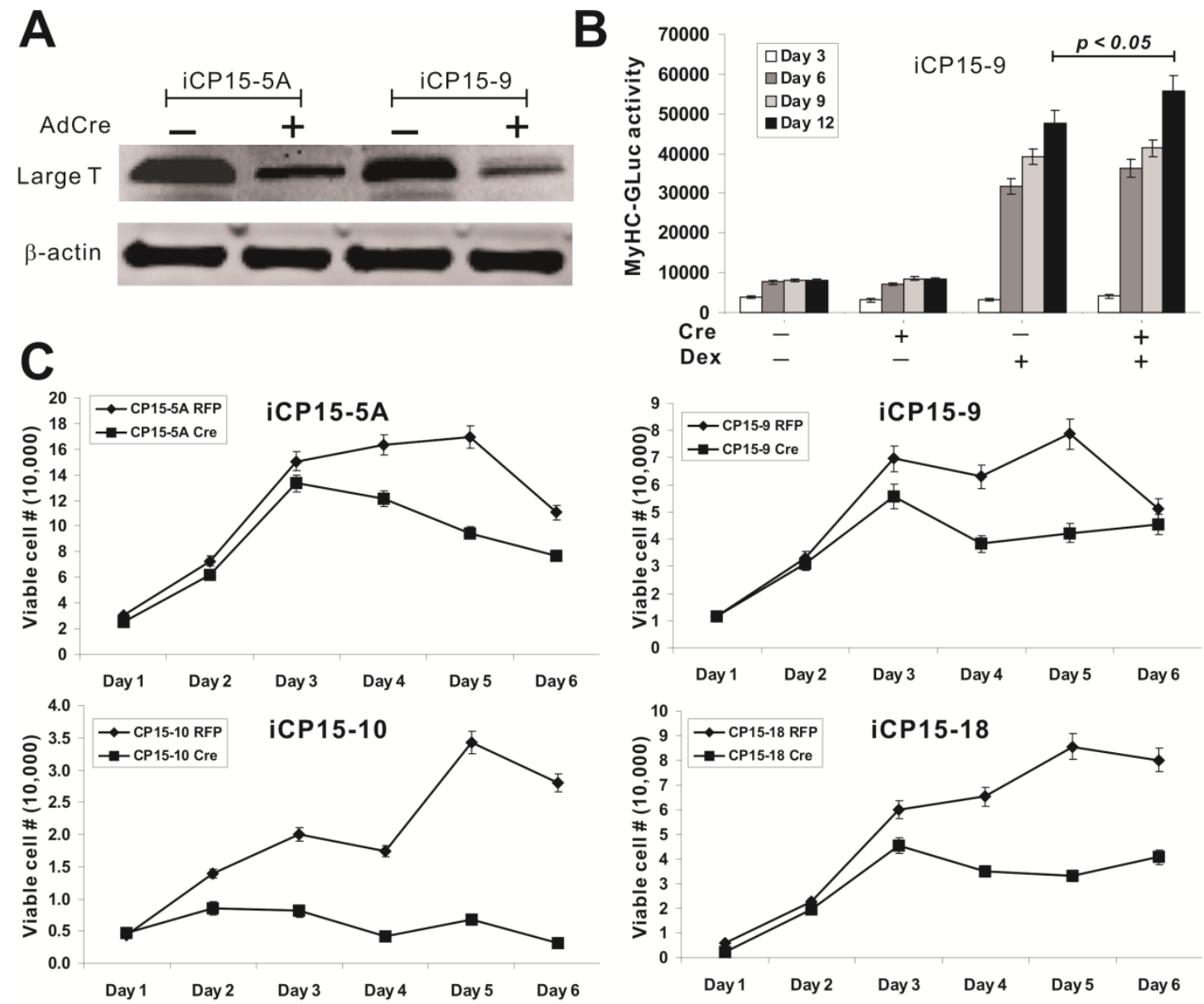

Figure 6. Characterization of iCPI 5 clones upon the removal of SV40 large T. (A) Efficient removal of SV40 T by Cre recombinase. iCPI 5 cells were infected with AdCre (+ lanes) or AdGFP (- lanes). Total cell lysate was subjected to Western blotting with an anti-large T antibody. Anti- $\beta$-actin blotting was used as a loading control. (B) Removal of large T increases differential potential of iCPI5-9 cells. MyHC-GLuc tagged iCPI5-9 cells were infected with AdCre (+ lanes) or AdGFP (lanes) and stimulated with Dex (I $\mu \mathrm{M}$, + lanes) or DMSO (- lanes). GLuc activity was measured at the indicated time points. (C) Removal of large T decreases differential potential of iCPI5 cells. Four iCPI5 clones were plated at subconfluence and infected with AdCre or AdRFP. Viable cells were counted at the indicated time points.

\section{DISCUSSION}

In this study, we investigate if mouse CPs derived from fetal hearts can be reversibly immortalized with SV40 large T antigen and maintain long-term cell proliferation without compromising cardiomyogenic differentiation potential. Using the system which ex- presses SV40 large T antigen flanked with LoxP sites, we demonstrate that mouse CPs derived from E15.5 fetal heart can be efficiently immortalized by SV40 large $\mathrm{T}$ antigen. The iCP15 cells exhibit an enhanced proliferative activity and maintain long-term cell proliferation, which can be reversed by Cre recombinase. In addition, the iCP15 cells express most cardiomyo- 
genic markers and retain differentiation potential as they can undergo terminal differentiate into cardiomyctes under appropriate differentiation conditions. Nonetheless, the removal of SV40 large T increases the differentiation potential of iCPs.

Traditionally, two major approaches have been used to immortalize primary cells, including the serial passages of primary cells until they overcome their growth-crisis stage and the transformation of primary cells by overexpression of one or more oncogenes. The serial passage has been used to establish the Balb/3T3 cell line [37]. However, most of these established cell lines were hypotetraploid [37]. Furthermore, it is unclear if these transformed cells retain multi-lineage differentiation potential. Numerous studies have been recently carried out to induce the pluripotency of fibroblasts and cardiomyogenic progenitors through reprogramming stem cell differentiation capability with a set of defined factors, such as Oct3/4, Sox9, Klf4, and c-Myc [38-42] or small molecules [43]. Our results have demonstrated that the reversible immortalization strategy using SV40 large T antigen is an efficient approach to establishing long-term cell culture of primary $\mathrm{CPs}$ for basic and translational research.

The large $T$ antigen encoded by simian virus 40 (SV40) plays essential roles in the infection of permissive cells, leading to production of progeny virions, and in the infection of nonpermissive cells, leading to malignant transformation [44, 45]. Primary CPs are nonpermissive for SV40, and infection by wild-type SV40 leads to immortalization and transformation of a small percentage of infected cells. The ability of SV40 large $\mathrm{T}$ antigen to immortalize CPs is largely dependent on its ability to complex with p53 [46]. Thus, SV40 T antigen has become one of the most commonly used gene to immortalize primary mammalian cells. In this report, we demonstrate that the iCPs are not tumorigenic at least within 4 weeks of implantation in athymic nude mice (data not shown). The Cre-mediated reversal of the immortalization phenotypes should further enhance the safety profile of this strategy. Furthermore, it has been well-documented that SV40 $\mathrm{T}$ antigen-transformed cells are in general not tumorigenic [47-50]. We have recently demonstrated the efficiency and safety of this strategy by immortalizing mouse mesenchemal stem cells and mouse melanoblasts [51, 52]. Nonetheless, it is noteworthy that the Cre-mediated excision of SV40 T antigen is not footprintless as a significant portion of the retroviral vector, such as LTRs and packaging signal, remains integrated in the host genome.

The telomerase (TERT) is another commonly used gene for immortalization [53]. The telomerase complex maintains telomere length, which is required for an unlimited cellular proliferation. Telomerase is low or absent in normal human somatic cells. Telomerase expression is high in stem cells but reduced upon differentiation. Restoring telomerase activity in normal somatic cells can indefinitely prolong cellular life span, which may be associated with the acquisition of characteristics typical of cellular transformation [54]. Ectopic expression of the catalytic subunit of mouse telomerase (mTERT) confers a growth advantage to primary cells and facilitates their spontaneous immortalization by targeting TGF $\beta$ pathway [55]. A recent study indicated that ectopic expression of mouse telomerase catalytic subunit (mTERT) does not affect embryonic stem (ES) cell proliferation or differentiation in vitro, but protects ES cells against cell death during differentiation [56]. Nonetheless, it is noteworthy that several studies have demonstrated that TGF $\beta$ signaling plays an important role in regulating the progenitor state of cardiovascular cells [13, $57,58]$. Thus, while the efficiency and differentiation potential of TERT-immortalized CPs needs to be thoroughly investigated, the SV40 T-mediated CP immortalization may be more advantageous.

While our current study demonstrates that conditionally immortalized iCPs not only maintain long-term cell proliferation but also retain cardiomyogenic differentiation potential, further studies should be directed to investigate the in vivo behavior of these iCP15 cells in a mouse model of infarcted heart. As cardiomyogenic differentiation is a complicated, well-coordinated and heterogeneous process, it would be of interest to investigate if the iCP15 cells can differentiate the cells typical of primary myocardium (early proliferating cardiac cells, pacemaker-like cells, His-, Purkinje-like cells) and working myocardium (atrial and ventricular) are formed $[2,10]$ in vivo.

In conclusion, in order to overcome the challenges in maintaining sufficient CPs for in vitro and in vivo studies we demonstrate that $\mathrm{CPs}$ can be efficiently immortalized by SV40 T antigen. The reversibly immortalized iCP15 cells exhibit high proliferative activity and maintain long-term cell proliferation, which can be reversed by Cre recombinase. The iCP15 cells express cardiomyocyte markers and retain cardiomyogenic potential. The removal of SV40 large T increases the differentiation potential of iCPs. Nonetheless, the reported reversible SV40 T antigen-mediated immortalization represents an efficient and safe approach for establishing long-term culture of primary cardiomyogenic progenitors for basic and translational research. 


\section{SUPPLEMENTARY MATERIAL}

Additional File 1:

Table S1, Figure S1 and Figure S2.

http://www.medsci.org/v10p1035s1.pdf

Additional File 2:

Video-S1. Primary cardiomyogenic cells derived from mouse E15.5 fetal heart (day 3).

http://www.medsci.org/v10p1035s2.avi

Additional File 3:

Video-S2. Immortalized E15.5 cardiomyogenic cells under hygromycin selection (day 7).

http://www.medsci.org/v10p1035s3.avi

\section{ABBREVIATIONS}

$\mathrm{CP}$, cardiomyogenic progenitor; cTnT, cardiac Troponin T; iCP, immortalized cardiomyogenic progenitor; MyHC, $\alpha$-myosin heavy chain.

\section{ACKNOWLEDGEMENTS}

We thank Dr. Jeffrey Robbins of the University of Cincinnati Children's Hospital Medical Center, Cincinnati, $\mathrm{OH}$ for generous provision of $\alpha-5.5$ vector ( $\alpha-\mathrm{MyHC}$ reporter plasmid). We are also grateful for Dr. Philippe Leboulch of Harvard Medical School for provision of the SSR \#69 vector. The reported work was supported in part by research grants from the Natural Science Foundation of China (Grant \#81100309 to YB and \#81001197 to YS), the Chongqing Science and Technology Commission (CSTC-2010BB5107 to GHZ), and the National Institutes of Health (TCH and HHL).

\section{COMPETING INTERESTS}

The authors have declared that no competing interest exists.

\section{REFERENCES}

1. Filipczyk AA, Passier R, Rochat A, Mummery CL. Regulation of cardiomyocyte differentiation of embryonic stem cells by extracellular signalling. Cell Mol Life Sci. 2007; 64: 704-18.

2. Perino MG, Yamanaka S, Li J, Wobus AM, Boheler KR. Cardiomyogenic stem and progenitor cell plasticity and the dissection of cardiopoiesis. J Mol Cell Cardiol. 2008; 45: 475-94.

3. Dowell JD, Rubart M, Pasumarthi KB, Soonpaa MH, Field LJ. Myocyte and myogenic stem cell transplantation in the heart. Cardiovasc Res. 2003; 58: 336-50.

4. Menasche P, Hagege AA, Scorsin M, Pouzet B, Desnos M, Duboc D, et al. Myoblast transplantation for heart failure. Lancet. 2001; 357: 279-80.

5. Menasche P, Hagege AA, Vilquin JT, Desnos M, Abergel E, Pouzet B, et al. Autologous skeletal myoblast transplantation for severe postinfarction left ventricular dysfunction. Journal of the American College of Cardiology. 2003; 41: 1078-83

6. Badorff C, Brandes RP, Popp R, Rupp S, Urbich C, Aicher A, et al. Transdifferentiation of blood-derived human adult endothelial progenitor cells into functionally active cardiomyocytes. Circulation. 2003; 107: 1024-32.

7. Hodgson DM, Behfar A, Zingman LV, Kane GC, Perez-Terzic C, Alekseev AE, et al. Stable benefit of embryonic stem cell therapy in myocardial infarction. American journal of physiology. 2004; 287: H471-9.
8. Orlic D, Kajstura J, Chimenti S, Jakoniuk I, Anderson SM, Li B, et al. Bone marrow cells regenerate infarcted myocardium. Nature. 2001; 410: 701-5.

9. Pittenger MF, Martin BJ. Mesenchymal stem cells and their potential as cardiac therapeutics. Circ Res. 2004; 95: 9-20.

10. Reffelmann T, Konemann S, Kloner RA. Promise of blood- and bone marrow-derived stem cell transplantation for functional cardiac repair: putting it in perspective with existing therapy. Journal of the American College of Cardiology. 2009; 53: 305-8.

11. Boheler KR, Czyz J, Tweedie D, Yang HT, Anisimov SV, Wobus AM. Differentiation of pluripotent embryonic stem cells into cardiomyocytes. Circ Res. 2002; 91: 189-201.

12. Kodama $H$, Inoue $T$, Watanabe R, Yasuoka H, Kawakami $Y$, Ogawa S, et al. Cardiomyogenic potential of mesenchymal progenitors derived from human circulating CD14+ monocytes. Stem Cells Dev. 2005; 14: 676-86.

13. Goumans MJ, de Boer TP, Smits AM, van Laake LW, van Vliet P, Metz $\mathrm{CH}$, et al. TGF-beta1 induces efficient differentiation of human cardiomyocyte progenitor cells into functional cardiomyocytes in vitro. Stem cell research. 2007; 1: 138-49.

14. Smits AM, van Laake LW, den Ouden K, Schreurs C, Szuhai K, van Echteld CJ, et al. Human cardiomyocyte progenitor cell transplantation preserves long-term function of the infarcted mouse myocardium. Cardiovasc Res. 2009; 83: 527-35.

15. Luo J, Tang M, Huang J, He BC, Gao JL, Chen L, et al. TGFbeta/BMP type I receptors ALK1 and ALK2 are essential for BMP9-induced osteogenic signaling in mesenchymal stem cells. J Biol Chem. 2010; 285: 29588-98.

16. Rastegar F, Gao JL, Shenaq D, Luo Q, Shi Q, Kim SH, et al. Lysophosphatidic acid acyltransferase beta (LPAATbeta) promotes the tumor growth of human osteosarcoma. PloS one. 2010; 5: e14182.

17. Sharff KA, Song WX, Luo X, Tang N, Luo J, Chen J, et al. Hey1 Basic Helix-Loop-Helix Protein Plays an Important Role in Mediating BMP9-induced Osteogenic Differentiation of Mesenchymal Progenitor Cells. J Biol Chem. 2009; 284: 649-59.

18. Su Y, Wagner ER, Luo Q, Huang J, Chen L, He BC, et al. Insulin-like growth factor binding protein 5 suppresses tumor growth and metastasis of human osteosarcoma. Oncogene. 2011; 30: 3907-17.

19. Zhang W, Deng ZL, Chen L, Zuo GW, Luo Q, Shi Q, et al. Retinoic acids potentiate BMP9-induced osteogenic differentiation of mesenchymal progenitor cells. PloS one. 2010; 5: e11917.

20. Westerman KA, Leboulch P. Reversible immortalization of mammalian cells mediated by retroviral transfer and site-specific recombination. Proc Natl Acad Sci U S A. 1996; 93: 8971-6.

21. Ng WA, Grupp IL, Subramaniam A, Robbins J. Cardiac myosin heavy chain mRNA expression and myocardial function in the mouse heart. Circ Res. 1991; 68: 1742-50.

22. He TC, Chan TA, Vogelstein B, Kinzler KW. PPARdelta is an APC-regulated target of nonsteroidal anti-inflammatory drugs. Cell. 1999; 99: 335-45.

23. He TC, Sparks AB, Rago C, Hermeking H, Zawel L, da Costa LT, et al. Identification of c-MYC as a target of the APC pathway [see comments]. Science. 1998; 281: 1509-12.

24. He TC, Zhou S, da Costa LT, Yu J, Kinzler KW, Vogelstein B. A simplified system for generating recombinant adenoviruses. Proc Natl Acad Sci US A. 1998; 95: 2509-14.

25. Luo J, Deng ZL, Luo X, Tang N, Song WX, Chen J, et al. A protocol for rapid generation of recombinant adenoviruses using the AdEasy system. Nat Protoc. 2007; 2: 1236-47.

26. Tang N, Song WX, Luo J, Luo X, Chen J, Sharff KA, et al. BMP9-induced osteogenic differentiation of mesenchymal progenitors requires functional canonical Wnt/beta-catenin signaling. J Cell Mol Med. 2009; 13: 2448-64.

27. He BC, Chen L, Zuo GW, Zhang W, Bi Y, Huang J, et al. Synergistic antitumor effect of the activated PPARgamma and retinoid receptors on human osteosarcoma. Clin Cancer Res. 2010; 16: 2235-45.

28. Bi Y, Huang J, He Y, Zhu GH, Su Y, He BC, et al. Wnt antagonist SFRP3 inhibits the differentiation of mouse hepatic progenitor cells. J Cell Biochem. 2009; 108: 295-303.

29. Huang J, Bi Y, Zhu GH, He Y, Su Y, He BC, et al. Retinoic acid signalling induces the differentiation of mouse fetal liver-derived hepatic progenitor cells. Liver Int. 2009; 29: 1569-81.

30. Zhu GH, Huang J, Bi Y, Su Y, Tang Y, He BC, et al. Activation of RXR and RAR signaling promotes myogenic differentiation of myoblastic C2C12 cells. Differentiation. 2009; 78: 195-204.

31. Luo Q, Kang Q, Si W, Jiang W, Park JK, Peng Y, et al. Connective Tissue Growth Factor (CTGF) Is Regulated by Wnt and Bone Morphogenetic Proteins Signaling in Osteoblast Differentiation of Mesenchymal Stem Cells. J Biol Chem. 2004; 279: 55958-68. 
32. Si W, Kang Q, Luu HH, Park JK, Luo Q, Song WX, et al. CCN1/Cyr61 Is Regulated by the Canonical Wnt Signal and Plays an Important Role in Wnt3A-Induced Osteoblast Differentiation of Mesenchymal Stem Cells. Mol Cell Biol. 2006; 26: 2955-64.

33. Peng Y, Kang Q, Luo Q, Jiang W, Si W, Liu BA, et al. Inhibitor of DNA binding/differentiation helix-loop-helix proteins mediate bone morphogenetic protein-induced osteoblast differentiation of mesenchymal stem cells. J Biol Chem. 2004; 279: 32941-9.

34. Brade T, Kumar S, Cunningham TJ, Chatzi C, Zhao X, Cavallero S, et al. Retinoic acid stimulates myocardial expansion by induction of hepatic erythropoietin which activates epicardial Igf2. Development. 2011; 138: 139-48.

35. Keegan BR, Feldman JL, Begemann G, Ingham PW, Yelon D. Retinoic acid signaling restricts the cardiac progenitor pool. Science. 2005; 307: 247-9.

36. Lin SC, Dolle P, Ryckebusch L, Noseda M, Zaffran S, Schneider MD, et al. Endogenous retinoic acid regulates cardiac progenitor differentiation. Proc Natl Acad Sci U S A. 2010; 107: 9234-9.

37. Aaronson SA, Todaro GJ. Development of 3T3-like lines from Balb-c mouse embryo cultures: transformation susceptibility to SV40. J Cell Physiol. 1968; 72: 141-8.

38. Takahashi K, Yamanaka S. Induction of pluripotent stem cells from mouse embryonic and adult fibroblast cultures by defined factors. Cell. 2006; 126: 663-76

39. Takahashi K, Tanabe K, Ohnuki M, Narita M, Ichisaka T, Tomoda K, et al. Induction of pluripotent stem cells from adult human fibroblasts by defined factors. Cell. 2007; 131: 861-72.

40. Okita K, Ichisaka T, Yamanaka S. Generation of germline-competent induced pluripotent stem cells. Nature. 2007; 448: 313-7.

41. Shi Y, Desponts C, Do JT, Hahm HS, Scholer HR, Ding S. Induction of pluripotent stem cells from mouse embryonic fibroblasts by Oct4 and Klf4 with small-molecule compounds. Cell stem cell. 2008; 3: 568-74.

42. Burridge PW, Keller G, Gold JD, Wu JC. Production of de novo cardiomyocytes: human pluripotent stem cell differentiation and direct reprogramming. Cell stem cell. 2012; 10: 16-28.

43. Pasha Z, Haider $H$, Ashraf M. Efficient non-viral reprogramming of myoblasts to stemness with a single small molecule to generate cardiac progenitor cells. PloS one. 2011; 6: e23667.

44. Borowiec JA, Dean FB, Bullock PA, Hurwitz J. Binding and unwinding--how T antigen engages the SV40 origin of DNA replication. Cell. 1990; 60: 181-4.

45. Prives $\mathrm{C}$. The replication functions of SV40 T antigen are regulated by phosphorylation. Cell. 1990; 61: 735-8.

46. Zhu JY, Abate M, Rice PW, Cole CN. The ability of simian virus 40 large $\mathrm{T}$ antigen to immortalize primary mouse embryo fibroblasts cosegregates with its ability to bind to p53. J Virol. 1991; 65: 6872-80.

47. Gee CJ, Harris H. Tumorigenicity of cells transformed by Simian virus 40 and of hybrids between such cells and normal diploid cells. J Cell Sci. 1979; 36: 223-40.

48. Howell N. Suppression of transformation and tumorigenicity in interspecies hybrids of human SV40-transformed and mouse 3T3 cell lines. Cytogenet Cell Genet. 1982; 34: 215-29.

49. Kahn P, Topp WC, Shin S. Tumorigenicity of SV40-transformed human and monkey cells in immunodeficient mice. Virology. 1983; 126: 348-60.

50. Nitta M, Katabuchi H, Ohtake H, Tashiro H, Yamaizumi M, Okamura H. Characterization and tumorigenicity of human ovarian surface epithelial cells immortalized by SV40 large T antigen. Gynecol Oncol. 2001; 81: 10-7.

51. Huang E, Bi Y, Jiang W, Luo X, Yang K, Gao JL, et al. Conditionally Immortalized Mouse Embryonic Fibroblasts Retain Proliferative Activity without Compromising Multipotent Differentiation Potential. PloS one. 2012; 7: e32428.

52. Yang K, Chen J, Jiang W, Huang E, Cui J, Kim SH, et al. Conditional Immortalization Establishes a Repertoire of Mouse Melanocyte Progenitors with Distinct Melanogenic Differentiation Potential. J Invest Dermatol. 2012; 132: 2479-83.

53. Counter CM, Hahn WC, Wei W, Caddle SD, Beijersbergen RL, Lansdorp $\mathrm{PM}$, et al. Dissociation among in vitro telomerase activity, telomere maintenance, and cellular immortalization. Proc Natl Acad Sci U S A. 1998; 95: 14723-8.

54. Kang MK, Park NH. Extension of cell life span using exogenous telomerase. Methods Mol Biol. 2007; 371: 151-65.

55. Geserick C, Tejera A, Gonzalez-Suarez E, Klatt P, Blasco MA. Expression of mTert in primary murine cells links the growth-promoting effects of telomerase to transforming growth factor-beta signaling. Oncogene. 2006; 25: 4310-9.

56. Lee MK, Hande MP, Sabapathy K. Ectopic mTERT expression in mouse embryonic stem cells does not affect differentiation but confers re- sistance to differentiation- and stress-induced p53-dependent apoptosis. J Cell Sci. 2005; 118: 819-29.

57. Goumans MJ, Liu Z, ten Dijke P. TGF-beta signaling in vascular biology and dysfunction. Cell Res. 2009; 19: 116-27.

58. Pardali E, Goumans MJ, ten Dijke P. Signaling by members of the TGF-beta family in vascular morphogenesis and disease. Trends Cell Biol. 2010; 20: 556-67 